\title{
Gene Expression Profiling and Identification of Resistance Genes to Aspergillus flavus Infection in Peanut through EST and Microarray Strategies
}

\author{
Baozhu Guo ${ }^{1}$, Natalie D. Fedorova ${ }^{2}$, Xiaoping Chen ${ }^{3,4}$, Chun-Hua Wan ${ }^{2}$, Wei Wang ${ }^{2}$, \\ William C. Nierman ${ }^{2,5}$, Deepak Bhatnagar ${ }^{6}$ and Jiujiang Yu ${ }^{6} *$
}

1 Crop Protection and Management Research Unit, U.S. Department of Agriculture-Agricultural Research Service, Tifton, GA 31794, USA; E-Mail: Baozhu.guo@ars.usda.gov

2

The J Craig Venter Institute, Rockville, MD 20850-3343, USA;

E-Mails: nfedorova@jcvi.org (N.D.F.); cwan@jcvi.org (C.-H.W.); wwang@jcvi.org (W.W.); wnierman@jcvi.org (W.C.N.)

3 Department of Plant Pathology, The University of Georgia, Tifton, GA 31793-0748, USA;

E-Mail: xpchen1011@gmail.com

4 Crops Research Institute, Guangdong Academy of Agricultural Sciences, Guangzhou 510640, China

5 Department of Biochemistry and Molecular Biology, The George Washington University School of Medicine, Washington, DC 20037, USA

6 Southern Regional Research Center, U.S. Department of Agriculture-Agricultural Research Service, New Orleans, LA 70124, USA; E-Mail: deepak.bhatnagar@ars.usda.gov

* Author to whom correspondence should be addressed; E-Mail: Jiujiang.yu@ ars.usda.gov;

Tel.: +1-504-286-4405; Fax: +1-504-286-4419.

Received: 27 April 2011; in revised form: 9 June 2011 / Accepted: 14 June 2011 /

Published: 24 June 2011

\begin{abstract}
Aspergillus flavus and A. parasiticus infect peanut seeds and produce aflatoxins, which are associated with various diseases in domestic animals and humans throughout the world. The most cost-effective strategy to minimize aflatoxin contamination involves the development of peanut cultivars that are resistant to fungal infection and/or aflatoxin production. To identify peanut Aspergillus-interactive and peanut Aspergillus-resistance genes, we carried out a large scale peanut Expressed Sequence Tag (EST) project which we used to construct a peanut glass slide oligonucleotide microarray. The fabricated microarray represents over $40 \%$ of the protein coding genes in the peanut genome. For expression profiling, resistant and susceptible peanut cultivars were infected with a mixture of Aspergillus flavus and parasiticus spores. The subsequent microarray analysis identified
\end{abstract}


62 genes in resistant cultivars that were up-expressed in response to Aspergillus infection. In addition, we identified 22 putative Aspergillus-resistance genes that were constitutively up-expressed in the resistant cultivar in comparison to the susceptible cultivar. Some of these genes were homologous to peanut, corn, and soybean genes that were previously shown to confer resistance to fungal infection. This study is a first step towards a comprehensive genome-scale platform for developing Aspergillus-resistant peanut cultivars through targeted marker-assisted breeding and genetic engineering.

Keywords: EST; microarray; gene profiling; peanut-fungus interaction; resistance genes; Aspergillus flavus; A. parasiticus; metarep

\section{Introduction}

Peanut (Arachis hypogaea L.) has been an important food and oil crop. Peanut contains not only a high percentage of oil (about 50\%) but also contains a high quality unsaturated fatty acid (oleic acid). These features confer superior oxidative stability for food products without further processing. Peanut oil is also low in saturated fat and rich in resveratrol, antioxidants, and other nutriceuticals, which may contribute to cardiovascular health. Currently, peanut is grown world-wide, predominantly in Asia, Africa, and North Americas, with about 21 million hectares under cultivation. World peanut production occupies an important role in the world economy with an estimated production value of about $\$ 35$ billion.

Research on the peanut genome is at an early stage. Major crop improvement emphasis is focused on using elite genetic stocks, cultural management, and disease and pest control measures to improve productivity and quality. Traditionally cultivar improvement has been limited by conventional breeding and selection strategies [1]. High throughput technologies such as whole genome and transcriptome sequencing and microarray analysis hold promise to greatly facilitate this process. To meet the needs of the peanut industry, the international research community developed the International Peanut Genomics Initiative to coordinate sequencing the complete peanut genome (http://www.peanutbioscience.com/peanutgenomeinitiative.html) [2,3]. Peanut is a polyploid organism with a large genome size $(2.8 \mathrm{~Gb})$, which makes whole genome sequencing prohibitively expensive. Furthermore, due to its polyploid nature, assembly, annotation, and analysis of the genome will be a very challenging task. Thus, alternative approaches such as Expressed Sequence Tag (EST) sequencing have been implemented to advance the understanding of the genome at a manageable cost.

Several research institutes have undertaken low to middle scale peanut Expressed Sequence Tag (EST) projects [4-6]. As early as 2005, Luo et al. [6] released the first batch of EST sequences from two cultivated peanut lines, which were later used to design the first peanut microarray $[7,8]$. Subsequently, our research group at the USDA reported a total 41,568 ESTs derived from Tifrunners and the breeding line GT-C20 [4,5]. Another group in Belgium generated 4847 ESTs from peanut mixed stages infected with the migratory peanut pod nematode [9]. A group at the University of Florida used suppression subtractive hybridization to identify differentially expressed ESTs from RKN-challenged root tissues in nematode-resistant and -susceptible peanut cultivars [10]. Lately, the 
Shandong Academy of Agricultural Sciences, China, has started a large scale EST project and has provided 17,000 expressed sequence tags (ESTs) [11].

With the increased awareness of aflatoxin contamination in peanut [2], the presence of aflatoxin in peanut products has become a serious food safety concern. It is a major financial concern to the peanut industry as more regulatory import measures take effect worldwide. Aflatoxin contamination in pre-harvested peanuts is caused by the infection of the Aspergillis species, mainly A. flavus and A. parasiticus. Understanding peanut-fungus interactions during the growth of both the peanut crop and the fungus is necessary to develop effective strategies to reduce or eliminate aflatoxin contamination of pre- and post-harvest peanut crop. Currently, peanut cultivars that are resistant to A. flavus and A. parasiticus infection are rare, and little is known about the molecular mechanisms that confer such resistance.

To gain a better understanding of these mechanisms, the USDA has initiated the peanut genome program [2]. We recently [12] developed and tested the utility of the first large-scale peanut microarray, investigating the gene expression in different peanut tissues such as pod, leaf, stem, root, and peg tissues. The study identified 108 putatively pod-specific/abundant genes [12]. Subsequently, as part of U.S. Peanut Genome Initiative supported by U.S. Industry and Peanut Growers, our group developed a large scale peanut EST project $[2,4,13]$ for the cultivated peanut and provided the genomic resources for use in marker development and gene discovery. Here we report the development of a peanut microarray based on these EST sequences as well as other publicly available peanut EST sequences down-loaded from dbEST database (NCBI, http://www.ncbi.nlm.nih.gov/) [14]. We employed this array in gene expression profiling experiments to identify candidate genes that confer resistance to Aspergillus infection due to up-expression in response to fungal infection using a resistant peanut line $v s$. a susceptible line.

\section{Materials and Methods}

\subsection{Peanut Lines Used}

Two peanut lines (cultivars) have been used in this experiment: Tifrunner and GT-C20, hereafter referred as C20. "Tifrunner" (TF) is a runner market-type peanut (Arachis hypogaea L. subsp. hypogaea var. hypogaea) cultivar with a high level of resistance to Tomato Spotted Wilt Virus (TSWV), moderate resistance to early (Cercospora arachidicola) and late leaf spot (Cercosporidium personatum), but it is a late maturity cultivar [15]. This cultivar is considered susceptible to Aspergillus infection in the field. "GT-C20" is a Spanish-type breeding line and highly susceptible to TSWV and leaf spots but resistant to aflatoxin contamination [16].

\subsection{Peanut Inoculation by Aspergillus during Growth}

Both resistant and susceptible peanut cultivars were subjected to infection with a mixture of A. flavus and A. parasiticus spores 60 days after planting (DAP). In order to mimic peanut field fungal population, A. parasiticus NRRL 2999 and A. flavus NRRL 3357 were used for inoculation because they are pre-dominant fungal strains in our peanut field. Peanut immature kernels were harvested 
30 days after inoculation. Total RNAs were isolated from these immature kernel seeds. Poly-A mRNAs were prepared from the total RNAs immediately prior to cDNA library construction.

\subsection{Expressed Sequence Tags and Sequencing}

Tissue collection, RNA isolation, cDNA library construction and sequencing were done at USDA-ARS, Crop Protection and Management Research Unit at Tifton, Georgia and US Horticultural Laboratory Genomics Research Center at Ft. Pierce, Florida. The peanut plant materials used for RNA extraction were grown in the field and inoculated at mid-bloom (60 DAP). Drought stress was imposed during the final 40 days before harvest through the use of rain-out shelters. Immature pods at the R5 (beginning seed), R6 (full seed) and R7 (beginning maturity) stages from "GT-C20" and "Tifrunner" were collected, frozen in liquid nitrogen, and stored at $-80{ }^{\circ} \mathrm{C}$ until RNA extraction. Leaf tissues were collected at 100 DAP under the natural occurrence of spotted wilt and leaf spot diseases of peanut genotypes, Tifrunner, GT-C20 and A13 [6,7]. Tissues were frozen in liquid nitrogen and stored at $-80{ }^{\circ} \mathrm{C}$ until RNA extraction by Trizol extraction. Tifrunner is resistant to TSWV and leaf spots, but susceptible to Aspergillus flavus. GT-C20 is susceptible to TSWV and leaf spots but resistant to A. flavus, and A13 (NCV11 $\times$ AR4) is moderately resistant to TSWV and leaf spots, and resistant to A. flavus infection [17].

EST libraries were constructed using the pBluescript ${ }^{\circledR}$ II XR cDNA Library Construction Kit (Stratagene, La Jolla, California, Catalog). Briefly, directional cDNA synthesis was made by attaching 5' EcoRI and 3' XhoI adaptors (oligo dT XhoI primer). After digesting with EcoRI and XhoI restriction enzymes, the cDNA inserts were ligated into the multicloning sites of pBluescript II SK (+) plasmid vector. The cDNAs in the pBluescript vector were sequenced using universal primers (5' T3 primer). Single pass, unidirectional (5' end) sequencing was performed using ABI 3730xl Genetic analyzer (Applied Biosystems) with the ABI Prism BigDye terminator cycle sequencing kit (Foster City, CA). Base calling was made using Phred and Trace Tuner (Paracel, Pasadena, CA, USA). The sequencing, sequence cleaning, end trimming, and assembly processing were performed in the Laboratory for Genomics and Bioinformatics, University of Georgia.

\subsection{Oligo Microarray Design}

The printed oligonucleotide sequences and the array platform description can be found at the NCBI GEO database (accession GPL13178). Briefly, oligonucleotides ranging from 60 to 70 mer were designed at the J. Craig Venter Institute (JCVI) and synthesized by Sigma-Aldrich (Saint Louis, MO). The total number of oligonucleotides spotted on the microarray was 6932, which represented 6932 peanut unigenes. They were spotted to Corning ultraGAPs glass slides with 3 replications of each oligonucleotide at different locations on the slide. With flip-dye hybridizations, the array platform generates 3 technical replications per hybridization.

\subsection{Microarray Experiment Design, Hybridization and Analysis}

Two factors were varied in the experimental design: peanut cultivars (TF and C20) and Aspergillus exposure. Combinations of these two factors allowed for four hybridization probe pairs for competitive hybridization as follows: 
- $\quad$ C20Y vs. TFY (GT-C20 infected vs. Tifrunner infected)

- $\quad$ C20Y vs. C20N (GT-C20 infected vs. not infected)

- $\quad$ TFY vs. TFN (Tifrunner infected vs. not infected)

- $\quad$ C20N vs. TFN (GT-C20 not infected vs. Tifrunner not infected)

The four samples were analyzed with four hybridizations each with a flip-dye control and three in-slide replicates as described (GEO records: GSM684493, GSM684512, and GSM684513).

\subsection{Data Processing for EST and Microarray Analysis}

Sequencing trace files from the cDNA peanut library were processed following the JCVI Sanger pipeline, which trims off vector and adaptor sequences and removes low-quality bases. Sequences sharing overlapping regions of greater than $94 \%$ identity over 40 or more continuous bases were assembled at high stringency using the CAP3 program and Paracel Transcript Assembler [18]; version 2.6.2, (http://www.paracel.com) [19] with modifications by the JCVI bioinformatics team. Overlaps based exclusively on low-complexity regions were excluded.

Hybridized slides were scanned using the standard protocol (see GEO records: GSM684493, GSM684512, and GSM684513 for details). All calculated gene expression ratios were $\log _{2}$-transformed and analyzed using MeV (http://www.tm4.org/mev.html) [20-22].

A gene was considered to be expressed if it had a positive expression value associated with it. $\log _{2}$ ratios were used to measure relative changes in expression level between two growth conditions. Genes were considered differentially expressed if the corresponding $\log _{2}$ ratios were greater than 2 . Gene Ontology (GO), enzyme classification (EC), and PFAM term enrichment analysis was performed using METAREP, an online annotation presentation tool developed at the JCVI (http://www.jcvi.org/metarep/dashboard/index) [23].

\section{Results and Discussion}

\subsection{Summary Classification of Expressed Sequence Tags(EST)}

A total of over 11,141 ESTs were assembled from over 100,000 Sanger reads generated in this study. Additional 2738 EST sequences were downloaded from the NCBI dbEST database including those sequences submitted by Shandong Academy of Agricultural Sciences. From this dataset, 13,879 unique ESTs (unigenes) have been assembled and annotated. The average GC content of these ESTs is $42.6 \%$ with the minimum GC of $15.8 \%$ and maximum GC of $72.5 \%$. It is estimated that the $2.8-\mathrm{Gb}$ peanut genome hosts 25,000-35,000 protein-coding genes, therefore 13,879 ESTs represent over 40\% of these genes. BLASTp search against the NCBI NR database showed that 1761 ESTs (12.7\%) can be assigned a putative function based on sequence similarity to previously characterized proteins. However $87.3 \%$ of the ESTs (12,118 ESTs) did not have significant hits in the database and were annotated as "hypothetical". Major functional categories represented in this EST set are listed in Table 1. The EST sequence data have been submitted to the NCBI EST database (ES702769 to ES724546 and ES751523 to ES768453). 
Table 1. Classification of identified genes in peanut.

\begin{tabular}{ll}
\hline Category of Genes & Number of Genes \\
\hline Hypothetical proteins & 12,118 \\
Ribosomal protein & 131 \\
Lopprotein & 91 \\
Cupin & 54 \\
Ribulose bisphophate carboxylase & 36 \\
Oleisin & 33 \\
Conglutin & 32 \\
Photosystem I and II & 29 \\
Protease inhibitor/seed storage protein & 28 \\
Core histone & 25 \\
Ara H8 allergen/alergen & 25 \\
Ubiquitin-conjugating enzyme & 23 \\
Peptidases & 22 \\
Epoxide hydrolase & 19 \\
Ras family protein & 16 \\
Glutathionine S-transferase & 16 \\
Zinc figure protein & 14 \\
Seed maturation protein & 13 \\
NAD/NADH dehydrogenase & 12 \\
Mem brane protein & 12 \\
Hsp20 & 11 \\
Peroxidase & 10 \\
14-3-3 protein & 10 \\
Universal stress protein & 9 \\
Oxidoreductase & 9 \\
HMG(high mobility group) box & 8 \\
Protein kinase & 6 \\
Polygalacturonase & 4 \\
Other & 1063 \\
\hline
\end{tabular}

\subsection{Identification of Resistant Genes to Aspergillus Infection Using Microarray Expression Data}

A 6932 gene-element oligonucleotide microarray was designed according to the 13,879 EST sequence information data set. Four microarray hybridizations were performed. We compared resistant peanut line, GT-C20, and susceptible peanut line, Tifrunner, under Aspergillus infected and non-infected conditions (C20Y vs. TFY; C20Y vs. C20N; C20N vs. TFN and TFY vs. TFN). The gene expression level is reported as $\log _{2}$ ratios of relative intensity. Among the 6932 genes whose RNA level was detected by the microarray, there were 401 genes that showed significant changes in gene expression level between resistant and susceptible peanut lines under infected and non-infected conditions. For each specific microarray hybridization, the number of up $\left(\log _{2} \geq 1.5\right)$ and down $\left(\log _{2} \leq-1.5\right)$ expressed genes are summarized in Table 2. It is interesting to find that there were a large number of genes in the resistant peanut line GT-C20 either highly or moderately up-expressed. Without Aspergillus infection (C20N vs. TFN), there were 9 and 31 genes in GT-C20 that scored as highly and moderately up-expressed compared with the susceptible line Tifrunner (C20N vs. TFN). 
With Aspergillus infection, the highly and moderately up-expressed genes were 25 and 40 respectively compared to the same strain without infection (C20Y vs. C20N). More interestingly, the resistant line, GT-C20, demonstrated a greater response to Aspergillus infection than the susceptible line Tifrunner (C20Y vs. TFY). The highly and moderately up-expressed genes were 52 and 126 respectively (C20Y vs. TFY). On the other hand, the susceptible line Tifrunner showed almost no response to Aspergillus infection (TFY vs. TFN). When under challenge by Aspergillus species, only one gene showed moderate up expression and four genes showed moderate down expression.

Table 2. Statistics of differentially expressed genes among 6932 expressed genes in peanut as detected by microarray.

\begin{tabular}{ccccc}
\hline & \multicolumn{4}{c}{ Differential Expression } \\
\hline Hybridizations & $\begin{array}{c}\text { Up-high } \\
\left(\mathbf{L o g}_{\mathbf{2}} \geq \mathbf{2}\right)\end{array}$ & $\begin{array}{c}\text { Up-mod } \\
\left(\mathbf{L o g}_{\mathbf{2}} \geq \mathbf{1 . 5} \mathbf{\&}<\mathbf{2}\right)\end{array}$ & $\begin{array}{c}\text { Down-high } \\
\left(\mathbf{L o g}_{\mathbf{2}} \leq-\mathbf{2}\right)\end{array}$ & $\begin{array}{c}\text { Down-mod } \\
\left(\mathbf{L o g}_{\mathbf{2}} \leq-\mathbf{1 . 5} \mathbf{\&}>\mathbf{- 2}\right)\end{array}$ \\
\hline C20Y vs. TFY & 52 & 126 & 51 & 99 \\
C20Y vs. C20N & 25 & 40 & 9 & 38 \\
C20N vs. TFN & 9 & 31 & 3 & 19 \\
TFY vs. TFN & 0 & 1 & 0 & 4 \\
\hline
\end{tabular}

Table 3 shows the 62 genes among the 178 up-expressed genes shown in Table 2 column 1 and column 2 (52 up-high and 126 up-mod) that were consistently highly up-expressed in response to Aspergillus infection in GT-C20 across two experiments (C20Y vs. TFY; C20Y vs. C20N) with expression levels significantly elevated $\left(\log _{2} \geq 1.5\right)$. While under non-infection condition, the expression levels are about the same as the susceptible line (C20N vs. TFN) (Table 3). Unfortunately, among the 62 expression elevated genes, only 8 genes were assigned biological functions based on their homologies to the corresponding genes in the database. The remaining 54 genes were classified as hypothetical proteins with no homologs in the existing database. From the consolidated data, we identified 22 genes in the resistant line (GT-C20) that were constitutively up-expressed compared with the susceptible line (Tifrunner) under infected (Table 4, C20Y vs. TFY, $\log _{2}$ values $\geq 1.5$ ) and non-infected conditions (Table $4, \mathrm{C} 20 \mathrm{~N}$ vs. TFN, $\log _{2}$ values $\geq 1.0$ ). Among the 22 genes, 5 genes showed slightly up-expression in response to Aspergillus infection compared with non-infection conditions (C20Y vs. C20N). Table 5 lists 42 genes in the resistant line GT-C20 that were consistently highly down-expressed in response to Aspergillus infection. Table 6 lists 24 genes in the resistant line GT-C20 that were constitutively down-expressed in the absence of infection or slightly down-expressed in response to Aspergillus infection (C20Y vs. TFY). 
Table 3. Peanut genes consistently highly expressed in response to fungal infection.

\begin{tabular}{|c|c|c|c|c|c|c|}
\hline Oligo Name & Locus IDs & $\begin{array}{c}\text { Annotation } \\
\text { Common Name }\end{array}$ & $\begin{array}{c}\text { C20Y } \\
\text { vs. } \\
\text { TFY }\end{array}$ & $\begin{array}{c}\mathrm{C} 20 \mathrm{Y} \\
\text { vs. } \\
\mathrm{C20N}\end{array}$ & $\begin{array}{c}\text { C20N } \\
\text { vs. } \\
\text { TFN }\end{array}$ & $\begin{array}{c}\text { TFY } \\
\text { vs. } \\
\text { TFN }\end{array}$ \\
\hline AH000387 & C20L-061_A09.ab1 & hypothetical protein & 3.16 & 1.43 & 0.17 & -0.53 \\
\hline AH001961 & gi|134038849 & hypothetical protein & 2.77 & 2.29 & 0.20 & -0.56 \\
\hline AH001521 & CL3249Contig1 & hypothetical protein & 2.72 & 1.84 & -0.04 & -0.07 \\
\hline AH000746 & C20L-034_H09.ab1 & hypothetical protein & 2.57 & 2.31 & -0.45 & -0.94 \\
\hline AH003951 & CL1062Contig1 & Cupin \| Oxalate oxidase & 2.54 & 2.42 & -0.27 & -0.22 \\
\hline AH006882 & CL1197Contig1 & hypothetical protein & 2.46 & 2.32 & -0.80 & -0.78 \\
\hline AH005123 & CL2491Contig1 & hypothetical protein & 2.44 & 2.27 & -0.84 & -0.75 \\
\hline AH007217 & SCL1Contig27 & hypothetical protein & 2.43 & 1.96 & 0.43 & -1.05 \\
\hline AH005015 & gi|56552992 & hypothetical protein & 2.43 & 2.49 & -0.63 & -0.85 \\
\hline AH000635 & CL647Contig1 & hypothetical protein & 2.39 & 2.38 & -0.19 & -0.50 \\
\hline AH002603 & CL129Contig1 & PA domain $\|$ Cucumisin & 2.38 & 2.26 & -0.90 & -1.11 \\
\hline AH002125 & gi|116359805 & hypothetical protein & 2.36 & 1.11 & 0.89 & -1.13 \\
\hline AH003731 & CL533Contig1 & hypothetical protein & 2.36 & 1.65 & -0.64 & -1.26 \\
\hline AH004262 & CL497Contig2 & hypothetical protein & 2.35 & 2.17 & 0.75 & -0.38 \\
\hline AH002955 & CL2001Contig1 & hypothetical protein & 2.35 & 2.37 & -0.10 & -0.43 \\
\hline AH001895 & CL282Contig1 & hypothetical protein & 2.34 & 1.23 & 0.83 & -0.57 \\
\hline AH002758 & C20L-064_H03.ab1 & hypothetical protein & 2.33 & 2.44 & -1.21 & -0.47 \\
\hline AH004570 & CL3695Contig1 & hypothetical protein & 2.30 & 2.43 & 0.37 & 0.42 \\
\hline AH006890 & CL1262Contig1 & $\begin{array}{l}\text { SCP-like extracellular } \\
\text { protein }\end{array}$ & 2.29 & 2.00 & -0.75 & -1.19 \\
\hline AH002029 & CL2422Contig1 & hypothetical protein & 2.28 & 1.12 & 0.02 & -0.91 \\
\hline AH001450 & CL3051Contig1 & hypothetical protein & 2.24 & 1.08 & 0.37 & -0.25 \\
\hline AH006106 & CL1112Contig1 & hypothetical protein & 2.22 & 1.97 & -0.13 & -0.24 \\
\hline AH002617 & gi|134037331 & hypothetical protein & 2.20 & 1.25 & 0.66 & -0.53 \\
\hline AH002238 & CL3Contig8 & hypothetical protein & 2.18 & 2.19 & -0.70 & -0.08 \\
\hline AH003578 & CL1337Contig1 & proline-rich protein & 2.16 & 2.01 & -1.09 & -0.09 \\
\hline AH002427 & CL516Contig1 & trypsin protein inhibitor 1 & 2.16 & 1.27 & 0.17 & -0.45 \\
\hline AH003300 & C20L-075_C10.ab1 & hypothetical protein & 2.13 & 1.45 & $\mathrm{NaN}$ & -1.10 \\
\hline AH000527 & gi|149223227 & hypothetical protein & 2.12 & 1.42 & 0.11 & 0.18 \\
\hline AH007516 & gi|149651508 & hypothetical protein & 2.09 & 2.09 & -0.93 & -0.68 \\
\hline AH000622 & CL818Contig1 & hypothetical protein & 2.08 & 1.91 & -0.60 & -0.51 \\
\hline AH004225 & CL2026Contig1 & hypothetical protein & 2.08 & 2.15 & -0.11 & -0.31 \\
\hline AH003174 & gi|115597367 & hypothetical protein & 2.05 & 1.45 & 0.47 & -0.39 \\
\hline AH001408 & CL1472Contig1 & hypothetical protein & 2.04 & 1.55 & -0.11 & -0.47 \\
\hline AH005389 & gi|116488586 & hypothetical protein & 2.02 & 1.45 & 0.56 & -0.74 \\
\hline AH006270 & CL433Contig1 & Protease inhibitor & 2.02 & 1.78 & 0.12 & -0.03 \\
\hline AH001007 & CL1820Contig1 & hypothetical protein & 2.01 & 1.58 & -0.49 & -0.55 \\
\hline AH007275 & gi|149648362 & hypothetical protein & 1.91 & 2.26 & -1.04 & 0.03 \\
\hline AH000272 & gi|149213703 & hypothetical protein & 1.91 & 1.03 & 0.07 & -0.37 \\
\hline AH006484 & CL2432Contig1 & hypothetical protein & 1.81 & 2.10 & 0.13 & 0.15 \\
\hline AH001135 & CL2410Contig1 & hypothetical protein & 1.81 & 1.31 & -0.41 & -0.44 \\
\hline
\end{tabular}


Table 3. Cont.

\begin{tabular}{|l|l|l|c|c|c|c|}
\hline AH002728 & gi|110813735 & hypothetical protein & 1.80 & 1.50 & 0.04 & -0.15 \\
\hline AH003280 & CL22Contig1 & hypothetical protein & 1.79 & 1.88 & -0.40 & -0.65 \\
\hline AH000855 & CL3205Contig1 & hypothetical protein & 1.79 & 1.26 & 0.58 & -0.58 \\
\hline AH004448 & $\begin{array}{l}\text { C20L-008-1- } \\
\text { T3_A01.ab1 }\end{array}$ & hypothetical protein & 1.78 & 1.48 & -0.25 & -0.28 \\
\hline AH000271 & CL6Contig3 & hypothetical protein & 1.76 & 1.89 & 0.00 & -0.32 \\
\hline AH003639 & CL919Contig1 & hypothetical protein & 1.76 & 1.02 & 0.23 & -0.25 \\
\hline AH004090 & CL1382Contig2 & hypothetical protein & 1.76 & 1.40 & 0.66 & 0.19 \\
\hline AH003402 & gi|116489695 & BURP domain & 1.76 & 1.63 & -0.18 & -0.43 \\
\hline AH001502 & CL44Contig1 & annexin & 1.74 & 1.09 & 0.47 & -0.54 \\
\hline AH007479 & gi|116489554 & hypothetical protein & 1.74 & 1.76 & -0.09 & -0.49 \\
\hline AH004337 & CL888Contig1 & hypothetical protein & 1.74 & 1.31 & 0.00 & -0.53 \\
\hline AH008308 & gi|134092873 & hypothetical protein & 1.72 & 1.78 & -0.25 & -0.54 \\
\hline AH003520 & CL844Contig2 & hypothetical protein & 1.71 & 1.07 & -0.63 & -1.13 \\
\hline AH002696 & CL3089Contig1 & hypothetical protein & 1.71 & 1.48 & -0.28 & -0.52 \\
\hline AH005990 & CL1886Contig1 & hypothetical protein & 1.70 & 1.74 & 0.23 & 0.09 \\
\hline AH003584 & CL1510Contig1 & hypothetical protein & 1.69 & 1.05 & -0.12 & -0.54 \\
\hline AH003682 & CL2193Contig1 & hypothetical protein & 1.63 & 2.15 & -1.03 & -0.04 \\
\hline AH002301 & gi|116488520 & hypothetical protein & 1.62 & 1.75 & -0.19 & 0.36 \\
\hline AH004952 & CL582Contig1 & hypothetical protein & 1.58 & 1.95 & -0.94 & -0.56 \\
\hline AH004306 & gi|134092818 & hypothetical protein & 1.57 & 1.33 & -0.16 & -0.51 \\
\hline AH005893 & gi|110810489 & hypothetical protein & 1.54 & 1.50 & -0.11 & 0.04 \\
\hline AH001402 & CL3953Contig1 & hypothetical protein & 1.52 & 2.08 & -0.14 & 0.30 \\
\hline
\end{tabular}

Note: The values are $\log _{2}$ ratios. For example, C20Y vs. TFY means $\log _{2}(\mathrm{C} 20 \mathrm{Y} / \mathrm{TFY})$. It is the expression level (RPKM) of resistant line GT-C20 compared with the susceptible line Tifrunner under Aspergillus infected condition ( $\mathrm{Y}$ ). The values are shaded red if $\geq 2$ and shaded yellow if the values are $\geq 1.5$ and $<2$. This applies to Tables $4,5,6$, and 7 . The negative values are shaded green if $\leq-2$ and shaded dark green if the values are $\leq-1.5$ and $>-2$.

Table 4. Resistant genes constitutively expressed.

\begin{tabular}{|c|l|l|c|c|c|c|}
\hline \multicolumn{1}{|c|}{$\begin{array}{c}\text { Oligo } \\
\text { Name }\end{array}$} & \multicolumn{1}{|c|}{ Locus IDs } & \multicolumn{1}{|c|}{$\begin{array}{c}\text { Annotation } \\
\text { common name }\end{array}$} & $\begin{array}{c}\text { C20Y } \\
\text { vs. } \\
\text { TFY }\end{array}$ & $\begin{array}{c}\text { C20Y } \\
\text { vs. } \\
\text { C20N }\end{array}$ & $\begin{array}{c}\text { C20N } \\
\text { vs. } \\
\text { TFN }\end{array}$ & $\begin{array}{c}\text { TFY } \\
\text { vs. } \\
\text { TFN }\end{array}$ \\
\hline AH003854 & CL974Contig1 & hypothetical protein & 2.51 & 0.23 & 1.17 & -1.34 \\
\hline AH004017 & $\begin{array}{l}\text { gi|116488752|gb| } \\
\text { EG529756.1 }\end{array}$ & hypothetical protein & 2.47 & 2.22 & 1.57 & -0.05 \\
\hline AH003192 & CL993Contig2 & 27K protein & 2.35 & 1.31 & 1.15 & -0.46 \\
\hline AH000555 & SCL3Contig5 & Cupin & 2.33 & 0.16 & 2.30 & 0.05 \\
\hline AH002179 & CL48Contig3 & $\begin{array}{l}\text { Delta(12)-fatty acid } \\
\text { dehydrogenase/desaturase }\end{array}$ & 2.27 & 1.22 & 1.66 & 0.09 \\
\hline AH002829 & CL432Contig1 & $\begin{array}{l}\text { Aminocyclopropanecarboxylate } \\
\text { oxidase }\end{array}$ & 2.24 & 0.26 & 1.28 & -1.44 \\
\hline AH006795 & CL2798Contig1 & hypothetical protein & 2.13 & 0.00 & 1.81 & -0.25 \\
\hline
\end{tabular}


Table 4. Cont.

\begin{tabular}{|c|c|c|c|c|c|c|}
\hline AH001501 & $\begin{array}{l}\text { gi }|147878026| \mathrm{gb} \mid \\
\text { ES538584.1 }\end{array}$ & $11 \mathrm{~S}$ seed storage globulin & 2.06 & 0.39 & 2.06 & 0.18 \\
\hline AH007210 & CL1250Contig1 & hypothetical protein & 1.99 & 1.07 & 1.26 & -0.13 \\
\hline AH004716 & $\begin{array}{l}\text { gi }|149650738| \mathrm{gb} \mid \\
\text { ES761721.1 }\end{array}$ & hypothetical protein & 1.96 & 1.40 & 1.39 & -0.18 \\
\hline AH000958 & CL101Contig5 & hypothetical protein & 1.95 & -0.66 & 1.91 & -0.32 \\
\hline AH002424 & $\begin{array}{l}\text { gi }|56690332| \mathrm{gb} \mid \\
\text { CX128235.1 }\end{array}$ & $\begin{array}{l}\text { NAD-specific glutamate } \\
\text { dehydrogenase (NAD-GDH) }\end{array}$ & 1.95 & 0.24 & 1.05 & -0.76 \\
\hline AH003909 & $\begin{array}{l}\text { gi }|110815482| \mathrm{gb} \mid \\
\text { EE126718.1 }\end{array}$ & hypothetical protein & 1.91 & 0.36 & 1.03 & -0.45 \\
\hline AH004786 & $\begin{array}{l}\text { gi }|116360311| \mathrm{gb} \mid \\
\text { EG374116.1 }\end{array}$ & Lipoxygenase & 1.90 & -0.15 & 1.94 & -0.62 \\
\hline AH003949 & $\begin{array}{l}\text { gi }|110815082| \mathrm{gb} \mid \\
\text { EE125141.1 }\end{array}$ & hypothetical protein & 1.85 & 0.31 & 1.20 & -1.03 \\
\hline AH002737 & CL121Contig1 & Lipoxygenase & 1.82 & -0.20 & 1.69 & -0.53 \\
\hline AH000129 & CL1899Contig1 & hypothetical protein & 1.77 & -0.11 & 1.86 & -0.03 \\
\hline AH002636 & CL101Contig4 & Cupin & 1.75 & -0.67 & 1.88 & -0.22 \\
\hline AH006951 & CL3246Contig1 & $\begin{array}{l}\text { cytochrome P450 } \\
\text { monooxygenase A16 \| } \\
\text { Ent-kaurene oxidase }\end{array}$ & 1.73 & -0.21 & 1.31 & -0.74 \\
\hline AH006650 & CL1140Contig1 & hypothetical protein & 1.54 & -0.46 & 1.07 & -0.67 \\
\hline AH003334 & $\begin{array}{l}\text { gi }|5726638| \mathrm{gb} \mid \mathrm{A} \\
\text { F172728.1| }\end{array}$ & hypothetical protein & 1.53 & -0.02 & 1.25 & -0.18 \\
\hline AH000421 & $\begin{array}{l}\text { gi }|115597155| \mathrm{gb} \mid \\
\text { EG029503.1 }\end{array}$ & hypothetical protein & 1.51 & 0.24 & 1.17 & -0.03 \\
\hline
\end{tabular}

Table 5. Consistently down expressed genes in response to fungal infection.

\begin{tabular}{|l|l|l|c|c|c|c|}
\hline \multicolumn{1}{|c|}{$\begin{array}{c}\text { Oligo } \\
\text { Name }\end{array}$} & \multicolumn{1}{|c|}{ Locus IDs } & \multicolumn{1}{|c|}{$\begin{array}{c}\text { Annotation } \\
\text { common name }\end{array}$} & $\begin{array}{c}\text { C20Y } \\
\text { vs. } \\
\text { TFY }\end{array}$ & $\begin{array}{c}\text { C20Y } \\
\text { vs. } \\
\text { C20N }\end{array}$ & $\begin{array}{c}\text { C20N } \\
\text { vs. } \\
\text { TFN }\end{array}$ & $\begin{array}{c}\text { TFY } \\
\text { vs. } \\
\text { TFN }\end{array}$ \\
\hline AH000690 & gi|149218418 & Gamma-thionin family & -1.51 & -1.71 & 1.12 & 0.43 \\
\hline AH002952 & CL1612Contig1 & hypothetical protein & -1.53 & -1.29 & -0.13 & 0.11 \\
\hline AH000772 & gi|110815456 & hypothetical protein & -1.53 & -1.08 & 0.05 & 0.59 \\
\hline AH007452 & CL3906Contig1 & hypothetical protein & -1.55 & -1.11 & 0.21 & 0.60 \\
\hline AH006152 & CL3978Contig1 & hypothetical protein & -1.57 & -1.59 & 0.15 & NaN \\
\hline AH000203 & C20L-061_H10.ab1 & hypothetical protein & -1.60 & -1.45 & -0.26 & 0.20 \\
\hline AH000929 & gi|110812895 & hypothetical protein & -1.66 & -1.16 & -0.93 & -0.03 \\
\hline AH006128 & gi|116489533 & CapLEA-2 & -1.72 & -2.37 & 2.01 & 0.73 \\
\hline AH005650 & CL45Contig1 & type 4 metallothionein & -1.72 & -1.97 & 1.58 & 0.90 \\
\hline AH000692 & gi|115596393 & hypothetical protein & -1.73 & -1.34 & 0.49 & 0.47 \\
\hline AH001026 & gi|110810654 & ethylene-responsive-binding & -1.73 & -1.57 & 0.11 & 0.42 \\
\hline AH008272 & CL558Contig1 & hypothetical protein & -1.79 & -1.26 & 0.63 & 0.47 \\
\hline AH008014 & gi|116360310 & hypothetical protein & -1.83 & -1.41 & 0.04 & 0.31 \\
\hline AH005128 & gi|134092758 & hypothetical protein & -1.83 & -1.05 & 0.37 & 0.35 \\
\hline
\end{tabular}


Table 5. Cont.

\begin{tabular}{|c|c|c|c|c|c|c|}
\hline AH003617 & CL2981Contig1 & lipid-transfer protein 3 (LTP 3) & -1.83 & -1.73 & 0.17 & 0.12 \\
\hline AH003051 & gi| 116360255 & A Lea protein & -1.87 & -1.58 & -0.19 & 0.54 \\
\hline AH007250 & gi|110812391 & hypothetical protein & -1.87 & -1.02 & 0.16 & 0.29 \\
\hline AH005235 & gi|110811079 & hypothetical protein & -1.90 & -1.94 & -0.37 & $\mathrm{NaN}$ \\
\hline AH005769 & CL45Contig2 & hypothetical protein & -1.90 & -1.90 & 1.15 & 0.79 \\
\hline AH003153 & C20L-030_F12.ab1 & hypothetical protein & -1.92 & -2.00 & 0.00 & $\mathrm{NaN}$ \\
\hline AH007709 & CL278Contig2 & seed maturation protein & -1.94 & -1.80 & 0.35 & 0.34 \\
\hline AH003511 & CL2196Contig1 & hypothetical protein & -1.95 & -1.29 & 0.14 & $\mathrm{NaN}$ \\
\hline AH005270 & gi|149655087 & hypothetical protein & -1.96 & -1.78 & -0.02 & -0.39 \\
\hline AH004182 & CL3386Contig1 & hypothetical protein & -1.96 & -1.66 & 0.00 & 0.43 \\
\hline AH007249 & C20L-073_B08.ab1 & hypothetical protein & -2.01 & -1.97 & 0.95 & -0.09 \\
\hline AH004352 & CL1037Contig1 & hypothetical protein & -2.03 & -1.47 & -0.64 & 0.09 \\
\hline AH007522 & gi|116488499 & hypothetical protein & -2.07 & -1.59 & -0.59 & 0.80 \\
\hline AH000339 & CL1492Contig1 & hypothetical protein & -2.10 & -1.67 & -0.11 & 0.74 \\
\hline AH004223 & gi|110810696 & hypothetical protein & -2.11 & -1.80 & 0.83 & 0.89 \\
\hline AH007290 & CL526Contig1 & protein binding & -2.14 & -1.40 & -0.39 & 0.57 \\
\hline AH006565 & gi|116488809 & hypothetical protein & -2.15 & -1.32 & -0.53 & -0.24 \\
\hline AH007623 & CL117Contig3 & hypothetical protein & -2.22 & -1.47 & 0.13 & 0.53 \\
\hline AH001324 & CL1130Contig1 & hypothetical protein & -2.25 & -1.51 & -0.30 & 0.61 \\
\hline AH005317 & CL117Contig1 & hypothetical protein & -2.34 & -1.65 & 0.39 & $\mathrm{NaN}$ \\
\hline AH003915 & gi|110813009 & hypothetical protein & -2.46 & -2.11 & 0.61 & $\mathrm{NaN}$ \\
\hline AH003052 & CL3662Contig1 & hypothetical protein & -2.52 & -1.26 & -0.25 & 0.32 \\
\hline AH003152 & CL953Contig1 & hypothetical protein & -2.75 & -1.96 & 0.00 & $\mathrm{NaN}$ \\
\hline AH008379 & CL139Contig1 & A Lea protein & -2.81 & -1.92 & -0.27 & 0.67 \\
\hline AH001493 & CL1974Contig1 & hypothetical protein & -2.88 & -2.45 & -0.88 & 0.17 \\
\hline AH005219 & CL890Contig2 & seed maturation protein PM22 & -2.90 & -1.97 & -0.50 & 0.88 \\
\hline AH006011 & CL3786Contig1 & hypothetical protein & -2.98 & -2.32 & 0.00 & 0.83 \\
\hline AH008363 & gi|149654533 & hypothetical protein & -3.17 & -2.16 & 0.00 & 1.09 \\
\hline
\end{tabular}

Table 6. Constitutively down expressed genes.

\begin{tabular}{|c|l|l|c|c|c|c|}
\hline $\begin{array}{c}\text { Oligo } \\
\text { Name }\end{array}$ & \multicolumn{1}{|c|}{ Locus IDs } & \multicolumn{1}{|c|}{$\begin{array}{c}\text { Annotation } \\
\text { common name }\end{array}$} & $\begin{array}{c}\text { C20Y } \\
\text { vs. } \\
\text { TFY }\end{array}$ & $\begin{array}{c}\text { C20Y } \\
\text { vs. } \\
\text { C20N }\end{array}$ & $\begin{array}{c}\text { C20N } \\
\text { vs. } \\
\text { TFN }\end{array}$ & $\begin{array}{c}\text { TFY } \\
\text { vs. } \\
\text { TFN }\end{array}$ \\
\hline AH005691 & gi|30419827 & hypothetical protein & -1.50 & 0.22 & -1.78 & -0.91 \\
\hline AH005600 & CL3445Contig1 & hypothetical protein & -1.52 & 0.15 & -1.05 & 0.50 \\
\hline AH004676 & CL3110Contig1 & hypothetical protein & -1.53 & -0.01 & -1.03 & -0.10 \\
\hline AH004895 & CL633Contig1 & hypothetical protein & -1.53 & -0.47 & -1.03 & -0.40 \\
\hline AH000806 & gi|134037244 & hypothetical protein & -1.54 & -0.51 & -1.05 & -0.34 \\
\hline AH008099 & gi|149653228 & hypothetical protein & -1.59 & 0.33 & -1.34 & 0.00 \\
\hline AH000790 & gi|134037353 & hypothetical protein & -1.73 & -0.47 & -1.18 & 0.07 \\
\hline AH004328 & CL633Contig2 & hypothetical protein & -1.77 & -0.71 & -1.86 & -1.08 \\
\hline AH003339 & gi|146771647 & hypothetical protein & -1.84 & 0.24 & -1.53 & 0.19 \\
\hline AH000324 & gi|110814436 & hypothetical protein & -1.92 & -0.49 & -1.58 & 0.12 \\
\hline
\end{tabular}


Table 6. Cont.

\begin{tabular}{|l|l|l|c|c|c|c|}
\hline AH002332 & C20L-052_A02.ab1 & hypothetical protein & -2.02 & -0.80 & -1.67 & 0.00 \\
\hline AH003062 & CL917Contig1 & hypothetical protein & -2.08 & -0.70 & -1.73 & -0.81 \\
\hline AH007482 & CL3870Contig1 & $\begin{array}{l}\text { P-enolpyruvate carboxykinase } \\
\text { (ATP) }\end{array}$ & -2.15 & -0.26 & -1.18 & 0.09 \\
\hline AH005991 & gi|110811967 & hypothetical protein & -2.17 & -0.51 & -1.27 & 0.00 \\
\hline AH003800 & gi|110812127 & hypothetical protein & -2.18 & -1.00 & -1.19 & 0.03 \\
\hline AH006388 & C20L-050_C02.ab1 & hypothetical protein & -2.29 & -0.31 & -1.58 & 0.16 \\
\hline AH005712 & C20L-069_A03.ab1 & hypothetical protein & -2.31 & -0.38 & -1.51 & 0.00 \\
\hline AH005618 & CL691Contig1 & hypothetical protein & -2.32 & 0.54 & -1.47 & 0.00 \\
\hline AH006196 & SCL3Contig23 & hypothetical protein & -2.38 & -0.51 & -1.73 & 0.08 \\
\hline AH007531 & C20L-055_H09.ab1 & hypothetical protein & -2.38 & -0.36 & -1.45 & 0.00 \\
\hline AH002687 & gi|72201444 & hypothetical protein & -2.60 & -0.91 & -1.77 & -0.16 \\
\hline AH000852 & CL3980Contig1 & hypothetical protein & -2.64 & -0.38 & -1.62 & 0.37 \\
\hline AH007569 & CL2822Contig1 & hypothetical protein & -2.74 & -0.15 & -1.68 & 0.23 \\
\hline AH006190 & CL488Contig3 & Thiosulfate sulfurtransferase & -3.08 & -0.37 & -2.47 & 0.00 \\
\hline
\end{tabular}

Table 7. GO biological processes of differentially expressed genes related to resistance.

\begin{tabular}{|c|c|c|c|c|c|c|}
\hline $\begin{array}{l}\text { Oligo } \\
\text { Name }\end{array}$ & Locus IDs & $\begin{array}{c}\text { Annotation } \\
\text { Common Name }\end{array}$ & $\begin{array}{c}\text { C20Y } \\
\text { vs. } \\
\text { TFY }\end{array}$ & $\begin{array}{c}\mathrm{C20Y} \\
\text { vs. } \\
\mathrm{C20N}\end{array}$ & $\begin{array}{c}\text { C20N } \\
\text { vs. } \\
\text { TFN }\end{array}$ & $\begin{array}{c}\text { TFY } \\
\text { vs. } \\
\text { TFN }\end{array}$ \\
\hline AH003951 & CL1062Contig1 & Cupin || Oxalate oxidase & 2.54 & 2.42 & -0.27 & -0.22 \\
\hline AH006890 & CL1262Contig1 & SCP-like extracellular protein & 2.29 & 2.00 & -0.75 & -1.19 \\
\hline AH002179 & CL48Contig3 & Fatty acid desaturase & 2.27 & 1.22 & 1.66 & 0.09 \\
\hline AH002829 & CL432Contig1 & $\begin{array}{l}\text { Aminocyclopropanecarboxylate } \\
\text { oxidase }\end{array}$ & 2.24 & 0.26 & 1.28 & -1.44 \\
\hline AH003578 & CL1337Contig1 & proline-rich protein & 2.16 & 2.01 & -1.09 & -0.09 \\
\hline AH002427 & CL516Contig1 & trypsin protein inhibitor 1 & 2.16 & 1.27 & 0.17 & -0.45 \\
\hline AH006270 & CL433Contig1 & $\begin{array}{l}\text { Protease inhibitor/seed } \\
\text { storage/LTP family }\end{array}$ & 2.02 & 1.78 & 0.12 & -0.03 \\
\hline AH004786 & gi|116360311 & Lipoxygenase & 1.90 & -0.15 & 1.94 & -0.62 \\
\hline AH001480 & CL3357Contig1 & polygalacturonase & 1.89 & 1.20 & 0.11 & -0.70 \\
\hline AH002965 & gi|146771622 & gibberellin regulated protein & 1.83 & 1.48 & 0.16 & -0.16 \\
\hline AH002737 & CL121Contig1 & Lipoxygenase || Lipoxygenase & 1.82 & -0.20 & 1.69 & -0.53 \\
\hline AH001196 & gi|115597159 & $\begin{array}{l}\text { Caffeate or } O \text {-diphenol- } O \text { - } \\
\text { methyl transferase }\end{array}$ & 1.80 & 0.53 & 0.34 & -0.79 \\
\hline AH006951 & CL3246Contig1 & $\begin{array}{l}\text { P450 monooxygenase A16 \| } \\
\text { Ent-kaurene oxidase }\end{array}$ & 1.73 & -0.21 & 1.31 & -0.74 \\
\hline AH004519 & CL3199Contig1 & $\begin{array}{l}\text { Protease inhibitor/seed } \\
\text { storage/LTP family }\end{array}$ & 1.35 & 1.83 & -0.49 & 0.12 \\
\hline AH002451 & CL2579Contig1 & lea protein 2 & -0.78 & -1.82 & 1.52 & 0.36 \\
\hline AH007902 & gi|149650530 & SCP-like extracellular protein & -1.60 & 0.71 & 0.00 & -0.94 \\
\hline AH006128 & gi|116489533 & $\begin{array}{l}\text { late embryogenesis abundant } \\
\text { protein } 2\end{array}$ & -1.72 & -2.37 & 2.01 & 0.73 \\
\hline AH005650 & CL45Contig1 & type 4 metallothionein & -1.72 & -1.97 & 1.58 & 0.90 \\
\hline
\end{tabular}


Table 7. Cont.

\begin{tabular}{|l|l|l|l|l|c|c|}
\hline AH001026 & gi|110810654 & $\begin{array}{l}\text { ethylene-responsive } \\
\text { element-binding protein }\end{array}$ & -1.73 & -1.57 & 0.11 & 0.42 \\
\hline AH003617 & CL2981Contig1 & $\begin{array}{l}\text { Non-specific lipid-transfer } \\
\text { protein 3 (LTP 3). }\end{array}$ & -1.83 & -1.73 & 0.17 & 0.12 \\
\hline AH003051 & gi|116360255 & A Lea protein & -1.87 & -1.58 & -0.19 & 0.54 \\
\hline AH004842 & CL2270Contig1 & auxin-responsive protein-related & -1.88 & -0.88 & 0.00 & 0.15 \\
\hline AH000878 & gi|110813054 & glyoxalase family protein & -2.18 & -1.35 & -0.52 & 0.60 \\
\hline AH004582 & gi|116488861 & glutathione S-transferase & -2.28 & -1.30 & 0.23 & 0.49 \\
\hline AH008379 & CL139Contig1 & A Lea protein & -2.81 & -1.92 & -0.27 & 0.67 \\
\hline
\end{tabular}

\subsection{Genes Resistant to Fungal Infection in Other Crop Systems have been Identified}

Among the genes whose putative biological functions have been postulated, we identified quite a few genes that were reportedly showing resistant to Aspergillus infection in other crop systems (Table 7). The trypsin protein inhibitor 1 (CL516Contig1) was demonstrated to be resistant to A. flavus infection in corn [24-27]. The lipoxygenase (CL121Contig1) also showed anti-fungal activities in peanut, corn, and soybean [28-31]. Several lines of evidence have indicated that lipoxygenase enzymes and their products, especially $9 \mathrm{~S}$ - and $13 \mathrm{~S}$-hydroperoxy fatty acids, could play a role in the Aspergillus/seed interaction. Both hydroperoxides exhibit sporogenic effects on Aspergillus spp. and differentially modulate aflatoxin pathway gene transcription.

Previous studies through gene cloning and characterization reported [28-32] the role of seed lipoxygenases, a peanut seed gene, PnLOX1. Analysis of its nucleotide sequence suggests that PnLOX1 encodes a predicted $98 \mathrm{kDa}$ protein highly similar in sequence and biochemical properties to soybean LOX2. The full-length PnLOX1 cDNA was subcloned into an expression vector to determine the type(s) of hydroperoxide products that the enzyme produces. Analysis of the oxidation products of PnLOX1 revealed that it produced a mixture of 30\% 9S-HPODE (9S-hydroperoxy-10E, 12Z-octadecadienoic acid) and 70\% 13S-HPODE (13S-hydroperoxy-9Z, 11E-octadecadienoic acid) at $\mathrm{pH}$ 7. PnLOX1 is an organ-specific gene which is constitutively expressed in immature cotyledons but is highly induced by methyl jasmonate, wounding, and Aspergillus infections in mature cotyledons. Examination of HPODE production in infected cotyledons suggests PnLOX1 expression may lead to an increase in 9S- HPODE in the seed [28-32]. The human lipoxigenase was also reported to degrade aflatoxin B1 by oxidative metabolism [33,34]. Those genes demonstrating resistance to fungal infection in other crops such as corn and soybean were also identified in peanut through this microarray gene profiling experiment. This result indicates that our data are consistent with previous studies in other crops and that this study provides new evidence for the roles of these proteins in protection against fungal infection.

\subsection{Defense-Related Genes Identified by Peanut Seed EST Database Search}

The EST sequences from "GT-C20" and "Tifrunner" were compared individually against peanut seed EST database. Among the EST sequences with $R>4$ [35], only three up-regulated putative defense-related genes were identified in both "GT-C20" and "Tifrunner" seed libraries. They were putative desiccation-related protein PCC13-62 precursor, serine protease inhibitor, and seed maturation 
protein LEA 4. Six up-regulated EST sequences were observed only in "GT-C20” seed EST libraries, and matched previous reported known proteins including PR10 protein, defensin protein, and calmodulin. In the "Tifrunner" seed EST libraries, five defense-related genes such as metallothioneinlike protein, heat shock protein and $\mathrm{Cu} / \mathrm{Zn}$ superoxide dismutase II exhibited significant up-regulation.

In the microarray experiments, several of the late embryo abundant (LEA) or late embryogenesis protein (LEA proteins) (CL2579Contig1 and CL139Contig1) were demonstrated highly or moderately down-expressed during fungal infection. The growth hormone genes for ethylene and auxin-responsive proteins (CL2270Contig1) were also down-expressed upon fungal infection. It is interesting to find that one of the SCP-like extracellular proteins was up-expressed (CL1262Contig1) while the other SCP-like extracellular proteins were down-expressed (ES761513.1|ES761513). The mechanisms of their expression in response to fungal infection deserves further investigation.

\section{Conclusions}

We described the sequence and assembly of 13,879 unique peanut ESTs, designed and constructed a 6932 gene-element oligonucleotide microarray, and analyzed the results of gene screening on the resistant genes in peanut in response to Aspergillus infection. More importantly, we identified resistant genes that are highly expressed in response to fungal infection. These genes could be valuable resources for follow-on research to transfer genes into commercial peanut cultivars through conventional breeding, marker assisted breeding, or through gene transfer by biotechnology. In addition, genetic regulation may be employed to boost the expression levels of these genes in the commercial cultivars to reduce or prevent aflatoxin contamination in peanut crop. EST and microarray technology has been demonstrated as robust in screening and identifying resistant or susceptible genes in large scale if not at the genome scale. Due to the lack of peanut whole genome sequence progress, the majority of the ESTs encode hypothetical proteins with unknown functions. We here demonstrate that using EST sequences and microarray strategies to screen and profile resistance genes provides a robust approach for identifying resistance genes and resistance gene candidates in the absence of a peanut genome sequence. Data presented in this report significantly identified gene targets for future crop improvement manipulation. Both the research methods and the resulting data will prove useful in crop improvement and aflatoxin contamination prevention.

\section{Acknowledgements}

We would like to thank Billy Wilson and Jake Fountain for technical assistance in the field and the laboratory. The sequencing was done at the U.S. Horticultural Laboratory, USDA-ARS, Fort Pierce, Florida. We thank Huiping Chen for assistance in cDNA library construction and clone preparation for sequencing and Charles Sun of JCVI for sequence clean up, assembly, and oligonucleotide and microarray design. We thank Yan Yu from the JCVI for her superb technical assistance for microarray printing. This research was supported by USDA Specific Cooperative Agreement 58-6602-6-121 with the University of Georgia, and partially supported by funds provided by Peanut Foundation and Georgia Agricultural Commodity Commission for peanut. Mention of trade names or commercial products in this publication is solely for the purpose of providing specific information and does not imply recommendation or endorsement by the U.S. Department of Agriculture. 


\section{References}

1. Guo, B.Z.; Chen, C.Y.; Chu, Y.; Holbrook, C.C.; Ozias-Akins, P.; Stalker, H.T. Advances in Genetics and Genomics for Sustainable Peanut Production. In Sustainable Agriculture and New Biotechnologies; Benkeblia, N., Ed.; CRC Press: Boca Raton, FL, USA, 2011, pp. 341-368.

2. Guo, B.Z.; Yu, J.; Holbrook, C.C.; Cleveland, T.E.; Nierman, W.C.; Scully, B.T. Strategy in prevention of prehavest aflatoxin contamination in peanuts: Aflatoxin biosynthesis, genetics and genomics. Peanut Sci. 2009, 36, 11-20.

3. Peanut Biosciences. Available on line: http://www.peanutbioscience.com/peanutgenomeinitiative.html (accessed on 23 June 2011).

4. Guo, B.; Chen, X.; Hong, Y.; Liang, X.; Dang, P.; Brenneman, T.; Holbrook, C.; Culbreath, A. Analysis of gene expression profiles in leaf tissues of cultivated peanuts and development of EST-SSR markers and gene discovery. Int. J. Plant Genomics 2009, 715605:1-715605:14.

5. Guo, B.; Chen, X.; Dang, P.; Scully, B.T.; Liang, X.; Holbrook, C.C.; Yu, J.; Culbreath, A.K. Peanut gene expression profiling in developing seeds at different reproduction stages during Aspergillus parasiticus infection. BMC Dev. Biol. 2008, 8, 12.

6. Luo, M.; Dang, P.; Guo, B.Z.; He, G.; Holbrook, C.C.; Bausher, M.G.; Lee, R.D. Generation of expressed sequence tags (ESTs) for gene discovery and marker development in cultivated peanut. Crop Sci. 2005, 45, 346-353.

7. Luo, M.; Dang, P.; Bausher, M.G.; Holbrook, C.C.; Lee, R.D.; Lynch, R.E.; Guo, B.Z. Identification of transcripts involved in resistance responses to leaf spot disease caused by cercosporidium personatum in peanut (Arachis hypogaea). Phytopathol. 2005, 95, 381-387.

8. Luo, M.; Liang, X.Q.; Dang, P.; Holbrook, C.C.; Bausher, M.G.; Lee, R.D.; Guo, B.Z. Microarray-based screening of differentially expressed genes in peanut in response to Aspergillus parasiticus infection and drought stress. Plant Sci. 2005, 169, 695-703.

9. Haegeman, A.; Jacob, J.; Vanholme, B.; Kyndt, T.; Mitreva, M.; Gheysen, G. Expressed sequence tags of the peanut pod nematode Ditylenchus africanus: the first transcriptome analysis of an Anguinid nematode. Mol. Biochem. Parasitol. 2009, 167, 32-40.

10. Tirumalaraju, S.V.; Jain, M.; Gallo, M. Differential gene expression in roots of nematode-resistant and -susceptible peanut (Arachis hypogaea) cultivars in response to early stages of peanut root-knot nematode (Meloidogyne arenaria) parasitization. J. Plant Physiol. 2011, 168, 481-492.

11. Bi, Y.P.; Liu, W.; Xia, H.; Su, L.; Zhao, C.Z.; Wan, S.B.; Wang, X.J. EST sequencing and gene expression profiling of cultivated peanut (Arachis hypogaea L.). Genome 2010, 53, 832-839.

12. Payton, P.; Kottapalli, K.R.; Rowland, D.; Faircloth, W.; Guo, B.; Burow, M.; Puppala, N.; Gallo, M. Gene expression profiling in peanut using high density oligonucleotide microarrays. BMC Genomics 2009, 10, 265.

13. Guo, B.Z.; Chen, X.P.; Dang, P.; Scully, B.T.; Liang, X.Q.; Holbrook, C.C.; Yu, J.; Culbreath, A.K. Peanut gene expression profiling in developing seeds at different reproduction stages during Aspergillus parasiticus infection. BMC Dev. Biol. 2008, 8, 12.

14. National Center for Biotechnology Information. Available online: http://www.ncbi.nlm.nih.gov (accesed on 23 June 2011).

15. Holbrook, C.C.; Culbreath, A.K. Registration of "Tifrunner" Peanut. J. Plant Regist. 2007, 1, 124. 
16. Liang, X.Q.; Holbrook, C.C.; Lynch, R.E.; Guo, B.Z. Beta-1,3-glucanase activity in peanut seed (Arachis hypogaea) is induced by inoculation with Aspergillus flavus and copurifies with a conglutin-like protein. Phytopathology 2005, 95, 506-511.

17. Mixon, A.C. Reducing Aspergillus species infection of peanut seed using resistant genotypes. J. Environ. Qual. 1986, 15, 101-103.

18. Huang, X.; Madan, A. CAP3: A DNA sequence assembly program. Genome Res. 1999, 9, 868-877.

19. Paracel BLAST. Available online: http://www.paracel.com (accessed on 23 June 2011).

20. Wilkinson, J.R.; Yu, J.; Abbas, H.K.; Scheffler, B.E.; Kim, H.S.; Nierman, W.C.; Bhatnagar, D.; Cleveland, T.E. Aflatoxin formation and gene expression in response to carbon source media shift in Aspergillus parasiticus. J. Food Addit. Contam. 2007, 24, 1051-1060.

21. Wilkinson, J.R.; Yu, J.; Bland, J.M.; Nierman, W.C.; Bhatnagar, D.; Cleveland, T.E. Amino acid supplementation reveals differential regulation of aflatoxin biosynthesis in Aspergillus flavus NRRL 3357 and Aspergillus parasiticus SRRC 143. Appl. Microbiol. Biotechnol. 2007, 74, 1308-1319.

22. Multi Experiment Viewer. Available online: http://www.tm4.org/mev.html (accessed on 23 June 2011).

23. J Craig Venter Institute Metagenomics Reports (METAREP). Available online: http://www.jcvi.org/metarep/dashboard/index (accessed on 23 June 2011).

24. Banks, W.A.; Niehoff, M.L.; Brown, R.L.; Chen, Z.Y.; Cleveland, T.E. Transport of an antifungal trypsin inhibitor isolated from corn across the blood-brain barrier. Antimicrob. Agents Chemother. 2002, 46, 2633-2635.

25. Chen, Z.Y.; Brown, R.L.; Lax, A.R.; Cleveland, T.E.; Russin, J.S. Inhibition of plant-pathogenic fungi by a corn trypsin inhibitor overexpressed in Escherichia coli. Appl. Environ. Microbiol. 1999, 65, 1320-1324.

26. Chen, Z.Y.; Brown, R.L.; Russin, J.S.; Lax, A.R.; Cleveland, T.E. A corn trypsin inhibitor with antifungal activity inhibits aspergillus flavus alpha-amylase. Phytopathology 1999, 89, 902-907.

27. Guo, B.Z.; Brown, R.L.; Lax, A.R.; Cleveland, T.E.; Russin, J.S.; Widstrom, N.W. Protein profiles and antifungal activities of kernel extracts from corn genotypes resistant and susceptible to Aspergillus flavus. J. Food Prot. 1998, 61, 98-102.

28. Burow, G.B.; Gardner, H.W.; Keller, N.P. A peanut seed lipoxygenase responsive to Aspergillus colonization. Plant Mol. Biol. 2000, 42, 689-701.

29. Burow, G.B.; Nesbitt, J.D.; Keller, N.P. Seed lipoxygenase products modulate Aspergillus mycotoxin synthesis. Mol. Plant Microbe Interact. 1997, 10, 380-387.

30. Wilson, R.A.; Gardner, H.W.; Keller, N.P. Cultivar-dependent expression of a maize lipoxygenase responsive to seed infesting fungi. Mol. Plant Microbe Interact. 2001, 14, 980-987.

31. Calvo, A.M.; Hinze, L.L.; Gardner, H.W.; Keller, N.P. Sporogenic effect of polyunsaturated fatty acids on development of Aspergillus spp. Appl. Environ. Microbiol. 1999, 65, 3668-3673.

32. Gao, X.; Brodhagen, M.; Isakeit, T.; Brown, S.H.; Gobel, C.; Betran, J.; Feussner, I.; Keller, N.P.; Kolomiets, M.V. Inactivation of the lipoxygenase ZmLOX3 increases susceptibility of maize to Aspergillus spp. Mol. Plant Microbe Interact. 2009, 22, 222-231.

33. Roy, S.K.; Kulkarni, A.P. Aflatoxin B1 epoxidation catalysed by partially purified human liver lipoxygenase. Xenobiotica 1997, 27, 231-241. 
34. Datta, K.; Kulkarni, A.P. Oxidative metabolism of aflatoxin B1 by lipoxygenase purified from human term placenta and intrauterine conceptal tissues. Teratology 1994, 50, 311-317.

35. Stekel, D.J.; Git, Y.; Falciani, F. The comparison of gene expression from multiple cDNA libraries. Genome Res. 2000, 10, 2055-2061.

(C) 2011 by the authors; licensee MDPI, Basel, Switzerland. This article is an open access article distributed under the terms and conditions of the Creative Commons Attribution license (http://creativecommons.org/licenses/by/3.0/). 